\title{
Photoluminescence dynamics of GaN under intense band-to-band and exciton resonant excitation
}

\section{$\operatorname{AUTHOR}(\mathrm{S})$ :}

Nagai, Takehiko; Yamamoto, Aishi; Kanemitsu, Yoshihiko

\section{CITATION:}

Nagai, Takehiko ... [et al]. Photoluminescence dynamics of GaN under intense band-toband and exciton resonant excitation. Physical Review B 2005, 71(12): 121201(R).

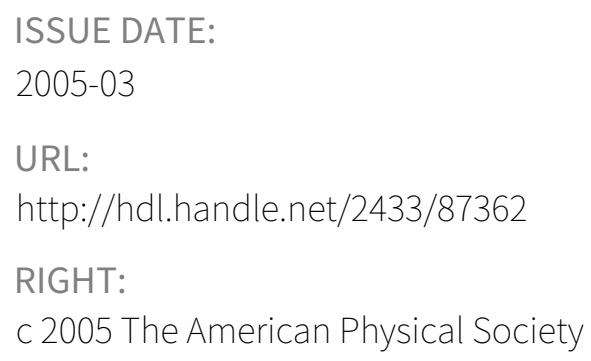


PHYSICAL REVIEW B 71, 121201(R) (2005)

\title{
Photoluminescence dynamics of GaN under intense band-to-band and exciton resonant excitation
}

\author{
Takehiko Nagai, ${ }^{1, *}$ Aishi Yamamoto, ${ }^{1}$ and Yoshihiko Kanemitsu ${ }^{2, \dagger}$ \\ ${ }^{1}$ Graduate School of Materials Science, Nara Institutes of Science and Technology, Ikoma, Nara 630-0192, Japan \\ ${ }^{2}$ International Research Center for Elements Science, Institute for Chemical Research, Kyoto University, Uji, Kyoto 611-0011, Japan
}

(Received 19 October 2004; revised manuscript received 16 December 2004; published 9 March 2005)

\begin{abstract}
We have studied the dynamics of highly dense exciton and electron-hole $(e-h)$ systems in GaN by means of optical Kerr-gate time-resolved photoluminescence (PL) spectral measurements. In high-density $e-h$ pairs above the Mott density, the broad electron-hole plasma emission appears at first, and then the narrow PL due to the biexciton and the inelastic exciton-exciton scattering is observed. When the pump light resonantly excites the $A$ excitons, highly dense $e-h$ systems with low effective temperatures are formed, and unique PL bands are observed. These PL peaks can be explained by a picture that the $A$ excitons are scattered into the $A, B$, and $C$ bands.
\end{abstract}

DOI: 10.1103/PhysRevB.71.121201

PACS number(s): 78.55.Cr, 71.35.Ee, 78.47.+p

Recently, the development of wavelength-tunable femtosecond laser systems and remarkable progress in semiconductor crystal growth have enabled us to study intrinsic optical processes and dynamics in wide-gap semiconductors. $\mathrm{GaN}$ and III-nitride compounds are key materials for fundamental semiconductor physics and also for blue and ultraviolet optoelectronic devices. ${ }^{1}$ Because of the large exciton binding energy, highly excited $\mathrm{GaN}$ provides us an excellent stage for the study of the excitonic many-body effects in semiconductors. With an increase of the excitation laser intensity, the exciton density exceeds the Mott transition density, and the exciton state turns to the electron-hole plasma (EHP) state. ${ }^{2}$ In highly excited GaN, the band-gap shrinkage and the broad EHP emission are observed because of the many-body effects in dense electron-hole $(e-h)$ systems. ${ }^{3-6}$ It is pointed out that in wide-gap $\mathrm{GaN}$ the $e-h$ pair correlation effect play an essential role in optical gains and electronic processes. $^{6-8}$ However, the dynamical behaviors of highly dense excitons and $e-h$ systems in GaN are not clear.

Time-resolved photoluminescence (PL) spectral measurements are one of the most useful methods for understanding the dynamics of highly dense excitons and $e-h$ systems. In $\mathrm{GaN}$ thin films, the PL dynamics have been studied only under the band-to-band excitation. ${ }^{3-6}$ Under the band-toband excitation, the photogenerated $e-h$ systems are "hot" because of large excess energy. On the other hand, the exciton resonant excitation produces "cold" $e-h$ systems with low effective carrier temperatures. ${ }^{9,10}$ The low-temperature and high-density $e-h$ systems provide essential information on the collective effects and excitonic processes in highly excited semiconductors. ${ }^{9-12}$ In this work, we report the PL dynamics of highly excited $\mathrm{GaN}$ at low temperatures, and the dynamical behaviors of highly dense excitons and $e-h$ systems are discussed through the comparison of time-resolved PL spectra between the band-to-band and the $A$-exciton resonant excitations. Under the exciton resonant excitation, unique exciton-related PL bands are clearly observed, and the origin of the PL bands is discussed.

The samples used in this work were fabricated by the metal organic chemical vapor deposition (MOCVD) method. The thickness of the GaN epitaxial layer was $5 \mu \mathrm{m}$, grown on a sapphire $c$-plane substrate with a $25-\mathrm{nm}$ GaN buffer layer. We determined that the transition energies of $A, B$, and $C$ excitons in our samples are 3.493, 3.502, and $3.516 \mathrm{eV}$, respectively, by means of photoreflectance measurements at 6 K. ${ }^{6}$

Wavelength-tunable femtosecond laser pulses were obtained from an optical parametric amplifier system based on a regenerative amplified mode-locked Ti:sapphire laser. The pulse duration and the repetition rate were $\sim 150 \mathrm{fs}$ and 1 $\mathrm{kHz}$, respectively. For time-resolved PL spectral measurements, an optical Kerr gate method was used with toluene as a Kerr medium in a quartz cell having 1-mm thickness. The time resolution was about $0.7 \mathrm{ps}$. The excitation photon energies for the band-to-band and the $A$-exciton resonant excitations were tuned to 3.723 and $3.493 \mathrm{eV}$, respectively. The PL spectra were measured as a function of the delay time, using a liquid-nitrogen-cooled charge-coupled device (CCD) with a $50-\mathrm{cm}$ single monochromator. The sample temperature was kept at $6 \mathrm{~K}$.

Figure 1 shows time-resolved PL spectra of GaN thin films under the band-to-band excitation $(3.723 \mathrm{eV})$ at different excitation intensities: (a) 8 , (b) 130 , and (c) $380 \mu \mathrm{J} / \mathrm{cm}^{2}$. Under weak excitation of $8 \mu \mathrm{J} / \mathrm{cm}^{2}$, a PL band appears at $3.486 \mathrm{eV}$. Similar PL bands appear under higher excitation intensities at several tens of picoseconds or more as shown in Figs. 1(b) and 1(c). The spectral shape of these PL bands can be fitted by an inverse-Boltzmann function, ${ }^{13}$ which is char-
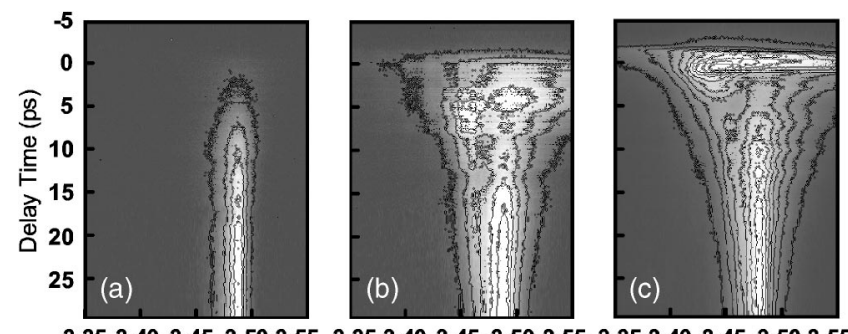

3.35 3.40 3.45 3.50 3.55 3.35 3.40 3.45 3.50 3.55 3.35 3.40 3.45 3.50 3.55 Photon Energy (eV)

FIG. 1. Time-resolved PL spectra of GaN thin films at $6 \mathrm{~K}$ under the band-to-band excitation $(3.723 \mathrm{eV})$. Excitation densities are (a) 8 , (b) 130 , and (c) $380 \mu \mathrm{J} / \mathrm{cm}^{2}$. 


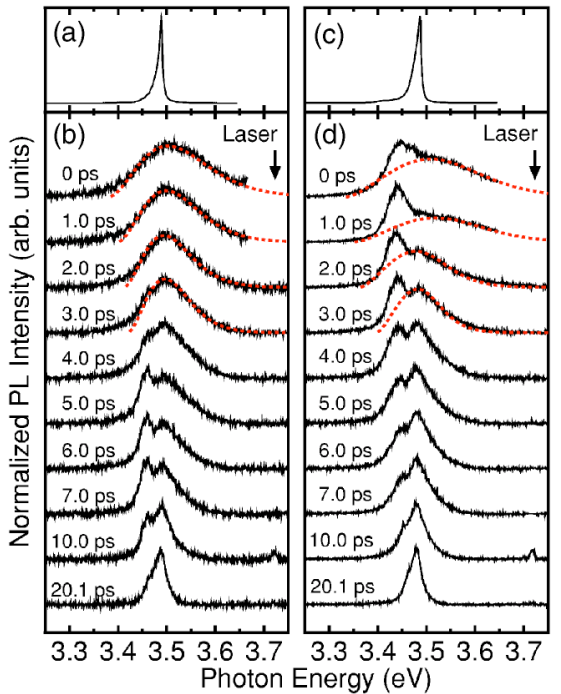

FIG. 2. (Color online) Temporal changes of the PL spectra of $\mathrm{GaN}$ thin films under the band-to-band excitation $(3.723 \mathrm{eV})$. The excitation densities are (a,b) 130 and $(\mathrm{c}, \mathrm{d}) 380 \mu \mathrm{J} / \mathrm{cm}^{2}$. Timeintegrated PL spectra are shown in (a) and (c). The broken curves are fitting using a non- $k$-conservation model.

acteristic for the spectral shape of the biexciton emission. In addition, the energy difference between the 3.486-eV PL peak and the 3.493-eV $A$-exciton peak is consistent with the biexciton binding energy of $\sim 6 \mathrm{meV}{ }^{14,15}$ Therefore, it is concluded that this PL band is due to the biexciton emission (so-called $M$ line). Because the exciton density is still high in the observed time region, a PL band due to the $A$ exciton was not observed.

Under high excitation intensities above $100 \mu \mathrm{J} / \mathrm{cm}^{2}$, the broad PL band appears just after the laser excitation, as shown in Figs. 1(b) and 1(c). The peak energy of the broad emission shifts towards lower energy with an increase of the excitation intensity. It is considered that the broad emission is attributed to the EHPs. The $M$-line emission appears around $3.486 \mathrm{eV}$ at $>5 \mathrm{ps}$. The appearance of the $M$ line suggests that the $e-h$ pair density rapidly decreases below the Mott density, and the exciton dynamics determines the PL properties. The EHP signal disappears rather fast ( $\sim 4 \mathrm{ps})$. The average lengths of the diffusion for electrons and holes at $t=4(\mathrm{ps})$ are $(D t)^{1 / 2}=100$ and $45 \mathrm{~nm}$, respectively, where $D$ is the diffusion coefficients, which are 25 and $5 \mathrm{~cm}^{2} / \mathrm{s}$ for electrons and holes for GaN, respectively. ${ }^{16}$ Since the penetration depth at $3.723 \mathrm{eV}$ is about $100 \mathrm{~nm}$, the decrease of electron-hole density by the diffusion is by a factor of $1 / 2$, and it is not negligible small. Consequently, the carrier diffusion process as well as radiative and nonradiative recombination of EHP causes the rapid disappearance of the EHP emission.

Figure 2 shows temporal changes of the PL spectra at $6 \mathrm{~K}$ under the band-to-band excitation: The excitation densities are $(\mathrm{a}, \mathrm{b}) 130$ and $(\mathrm{c}, \mathrm{d}) 380 \mu \mathrm{J} / \mathrm{cm}^{2}$. The time-integrated PL spectra are shown in Figs. 2(a) and 2(c). It is known that the spectral shape of spontaneous EHP emission can be described by the non- $k$-conservation model, where the $k$-selection rule for optical transitions is assumed to be bro-

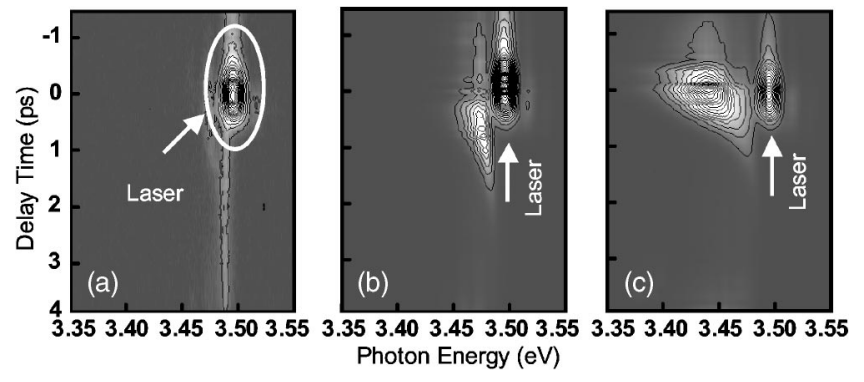

FIG. 3. Time-resolved PL spectra of GaN thin films at $6 \mathrm{~K}$ under the $A$-exciton resonant excitation $(3.493 \mathrm{eV})$. Excitation densities are (a) 7 , (b) 110 , and (c) $330 \mu \mathrm{J} / \mathrm{cm}^{2}$.

ken in highly dense $e$ - $h$ systems. ${ }^{17,18}$ In this calculation, the spectral shape of the EHPs is determined by the renormalized band-gap energy $\left(\widetilde{E_{g}}\right)$, the $e-h$ pair density $\left(n_{e-h}\right)$, and the effective electron temperature $\left(T_{e}\right)$. The experimental data at short delay times within 3 ps can be fitted very well by this model, shown by the broken curves in Figs. 2(b) and $2(\mathrm{~d}) .{ }^{19}$ When the excitation density is $380 \mu \mathrm{J} / \mathrm{cm}^{2}, n_{e-h}$ estimated from the above-mentioned spectral shape calculation is $4 \times 10^{19} \mathrm{~cm}^{-3}$ at $0 \mathrm{ps}$. We also calculated it using absorption coefficient $\left(6 \times 10^{4} \mathrm{~cm}^{-1}\right)^{6}$ and reflectivity $(20 \%){ }^{20}$ The calculated value is $3.1 \times 10^{19} \mathrm{~cm}^{-3}$, which is almost the same as that we estimated from the spectral shape calculation. These results indicate that the broad emission is attributed to the spontaneous emission of EHPs. In addition, in Fig. 2(d), the narrow PL band is superimposed on the broad EHP emission within $\sim 3$ ps. This is due to the stimulated emission of EHPs, because the peak energy of the narrow superimposed PL band agrees with that of the optical gain spectrum. ${ }^{21}$

In Fig. 2(b), at the delay time between 5 and 10 ps, a peak appears below the $M$-line emission energy. It is believed that this emission is due to the exciton-exciton scattering (socalled $P$ line) emission, ${ }^{22}$ whose details will be discussed later. Under the band-to-band excitation, time-resolved PL studies show that the EHP emission appears just after the laser excitation and sequentially the exciton-related PL bands appear with delay time. However, the band-to-band excitation produces high-temperature $e-h$ systems. The thermal broadening due to high electron temperatures leads to ambiguous discussions. Rather, we can clearly observe the change of the EHP state to the exciton state and the excitonrelated PL in low-temperature $e-h$ systems formed by the $A$-exciton resonant excitation.

Figure 3 shows the time-resolved PL spectra under the $A$-exciton resonant excitation $(3.493 \mathrm{eV})$ at (a) 7 , (b) 110 , and (c) $330 \mu \mathrm{J} / \mathrm{cm}^{2}$. Under the $A$-exciton resonant excitation, the scattering of the laser light appears around $0 \mathrm{ps}$ delay time. In Fig. 3(a), the $M$ line at $3.486 \mathrm{eV}$ appears at the delay time of about 2 ps. With an increase of the excitation density, at around 0 ps delay time the broad PL band due to the EHPs appears at $\sim 3.45 \mathrm{eV}$, and the peak energy slightly shifts to the higher energy with delay time.

Figure 4 shows temporal changes of the PL spectra under the $A$-exciton resonant excitation at $330 \mu \mathrm{J} / \mathrm{cm}^{2}$. The integrated PL spectrum is shown in Fig. 4(a). With an increase of the delay time, the broad emission due to the EHPs continu- 


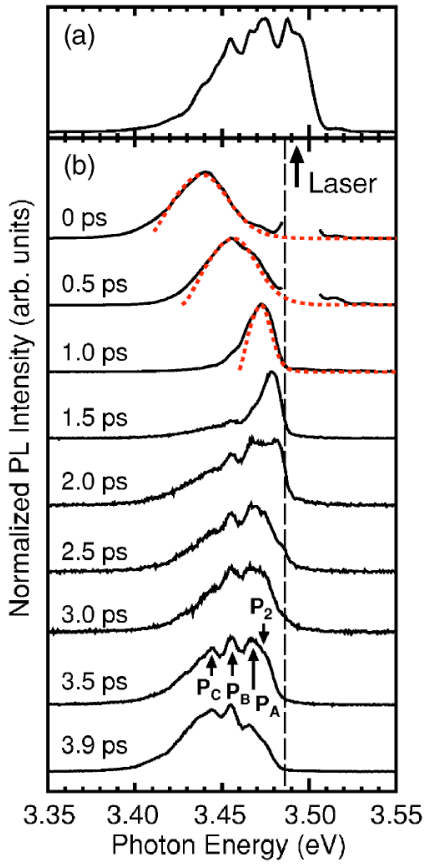

FIG. 4. (Color online) Temporal changes of PL spectra of GaN thin films under the $A$-exciton resonant excitation $(3.493 \mathrm{eV})$. The excitation density is $330 \mu \mathrm{J} / \mathrm{cm}^{2}$. Time-integrated PL spectra are shown in (a). The broken curves are fitting using a non$k$-conservation model.

ously shifts to the $M$-line peak energy, where the $M$-line energy is indicated by the dashed line in the figure. The broken curves are calculated spectra using the non$k$-conservation model. ${ }^{17,18}$ There is a good agreement between the experimentally observed and theoretically calculated PL spectra. Surprisingly, the spectral shape of the PL band drastically changes after $\sim 2$ ps, i.e., a few new PL peaks appear below the $M$-line energy. Three PL peaks appear at $3.468,3.456$, and $3.445 \mathrm{eV}$, and these are indicated by $P_{A}, P_{B}$, and $P_{C}$, respectively. In addition, we also observed a shoulder at higher energy of $P_{A}$ line, which is denoted by $P_{2}$. Since the EHP emission disappears at $\sim 3.5 \mathrm{ps}, n_{e-h}$ decreases below the critical density of the exciton Mott transition.

In extremely weak excitation condition, the PL band due to the bound exciton was observed. Since the density of the impurities is finite, the number of the bound excitons is saturated with increasing excitation density. Thus, free-excitonrelated PL bands, such as free-exciton, $M$ and $P$ lines, emerge. In highly dense exciton states, inelastic excitonexciton scattering processes contribute to the PL spectra. In inelastic exciton scattering processes, two $n=1$ excitons are scattered, and one of them is excited to higher energy exciton states such as $n=2,3, \ldots \infty$, while the other is scattered into a photonlike state. ${ }^{2}$ The emission due to the photonlike state appears at lower energy of $A$ exciton, which corresponds to the energy difference between the high-energy scattered exciton states and the $n=1$ exciton state. When the exciton is scattered into the $n=2 A$-exciton state, the $P$ line appears at $3.474 \mathrm{eV}$, where exciton binding energy is $25 \mathrm{meV} .{ }^{20}$ When the exciton is scattered into the $n=\infty$ state (i.e., the continu-

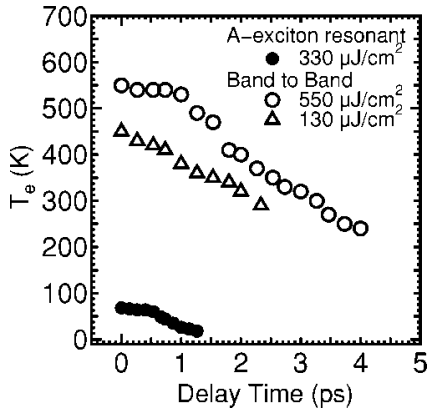

FIG. 5. Effective electron temperature $\left(T_{e}\right)$ as a function of the delay time. Open circles and triangles are under the band-to-band excitation, and solid circles are under the $A$-exciton resonant excitation. The excitation intensity is shown in the figure.

ous $A$ band), the $P$ line appears at $3.467 \mathrm{eV}$. These values agree with the energies of $P_{2}$ and $P_{A}$. We denote the scattering process for the $P_{A}$-line as $2 X_{A} \rightarrow A+P_{A}$, where the $A$-exciton state is indicated as $X_{A}$. In addition, there are two PL peaks $\left(P_{B}\right.$ and $\left.P_{C}\right)$ at the lower energy side. It is possible that the $n=1 A$ exciton is scattered into the other bands such as the $B$ and $C$ bands. If the binding energies of the $B$ and $C$ excitons are almost the same as that of the $A$ exciton, the peak energies of $P$ lines due to the scattering into the $B$ and $C$ bands are 3.458 and $3.444 \mathrm{eV}$. Here the energy differences between the $A$ and $B$ bands and between the $A$ and $C$ bands are 9 and $23 \mathrm{meV}$, respectively. These values are approximately consistent with the energies of the $P_{B}$ and $P_{C}$ bands. Hence, it is concluded that the $P_{B}$ and $P_{C}$ bands originate from the $P$ line due to the scattering of the $n=1 A$ exciton into the $B$ and $C$ bands. These processes can be expressed as $2 X_{A} \rightarrow B+P_{B}$ and $2 X_{A} \rightarrow C+P_{C}$, respectively. This is a clear observation of the inelastic scatterings of excitons into different bands in GaN. These PL bands are not resolved in the time-integrated PL spectrum of Fig. 4(a). By using timeresolved PL spectroscopy they are clearly distinguished. There is no theoretical study on the inelastic $A$-exciton scattering processes into other bands, and it is difficult to discuss the selection rules in highly dense exciton systems at present. This point is under discussion in this field. ${ }^{8}$

From the fitting of spontaneous EHPs emission spectra shown by the broken curves in Figs. 2(b), 2(d), and 4(b), effective electron temperature $T_{e}$ can be obtained. Figure 5 shows $T_{e}$ as a function of the delay time. The open circles and triangles show the $T_{e}$ at the excitation intensity of 550 and $130 \mu \mathrm{J} / \mathrm{cm}^{2}$ under the band-to-band excitation. The solid circles show the $T_{e}$ at $330 \mu \mathrm{J} / \mathrm{cm}^{2}$ under the $A$-exciton resonant excitation. Under the band-to-band excitation, $T_{e}$ rises to $\sim 500 \mathrm{~K}$ just after the laser excitation. On the other hand, $T_{e}$ is only $\sim 70 \mathrm{~K}$ under the $A$-exciton resonant excitation, and is much lower than that under the band-to-band excitation, because the excess energy of the excitation photon is small in the case of the resonant excitation. Moreover, under the $A$-exciton resonant excitation, the increase of the lattice temperature is smaller, and $T_{e}$ becomes $\sim 20 \mathrm{~K}$ after $\sim 1.5 \mathrm{ps}$, as shown in Fig. 5. When we estimated the $P$-line energy after $\sim 3.5$ ps in Fig. 4 , we assumed that the $T_{e}$ was 6 K. From Fig. 5, it is considered that this assumption is reasonable. Here, we should note that the exciton binding en- 
ergy is $25 \mathrm{meV}$, which means that exciton is stable below $\sim 290 \mathrm{~K}^{23}$ The temperature $T_{e}$ is well below it even at 0 ps under the resonant excitation. Therefore, highly dense and cold excitons were created under the resonant excitation, and PL lines, $P_{B}$ and $P_{C}$, were able to be observed. In the band-to-band excitation, however, the thermal broadening due to the high $T_{e}$ hinders the fine structures in the PL spectra.

In conclusion, we have studied the PL dynamics of highly dense $e-h$ and exciton systems near the Mott density under the band-to-band and the $A$-exciton resonant excitations in $\mathrm{GaN}$. Under the band-to-band excitation, the effective electron temperature is high, and broad electron-hole plasma and exciton-related emissions were observed. Under the $A$-exciton resonant excitation, the emissions due to inelastic scattering of the $n=1 A$ exciton into the $A, B$, and $C$ bands appeared after several picoseconds. The detailed optical processes in highly excited GaN were revealed by time-resolved PL spectra of cold $e$ - $h$ systems formed by the $A$-exciton resonant excitation.

The authors would like to thank Dr. H. Inouye for experimental assistance and Dr. T. J. Inagaki for useful discussions. This work was supported in part by a Grant-in-Aid for Scientific Research from the Japan Society for the Promotion of Science.
*Present address: Research Center for Photovoltaic Advanced Crystalline Silicon, National Institute of Advanced Industrial Science and Technology, Tsukuba, Ibaraki, 305-8567, Japan.

${ }^{\dagger}$ Author to whom correspondence should be addressed; Email address: kanemitu@scl.kyoto-u.ac.jp

${ }^{1}$ S. Nakamura, S. Pearton, and G. Fasol, The Blue Laser Diode (Springer, Berlin, 2000).

${ }^{2}$ C. F. Klingshirn, Semiconductor Optics (Springer, Berlin, 1997).

${ }^{3}$ See, for example, J. C. Holst, L. Eckey, A. Hoffmann, I. Broser, H. Amano, and I. Akasaki, MRS Internet J. Nitride Semicond. Res. 2, 25 (1997).

${ }^{4}$ J. Holst, L. Eckey, A. Hoffmann, I. Broser, B. Schöttker, D. J. As, D. Schikora, and K. Lischka, Appl. Phys. Lett. 72, 1439 (1998).

${ }^{5}$ K. Omae, Y. Kawakami, S. Fujita, Y. Kiyoku, and T. Mukai, Appl. Phys. Lett. 79, 2351 (2001).

${ }^{6}$ T. Nagai, T. J. Inagaki, and Y. Kanemitsu, Appl. Phys. Lett. 84, 1284 (2004).

${ }^{7}$ W. W. Chow, A. Knorr, and S. W. Koch, Appl. Phys. Lett. 67, 754 (1995)

${ }^{8}$ T. J. Inagaki and M. Aihara, Phys. Rev. B 65, 205204 (2002).

${ }^{9}$ G. W. Fehrenbach, W. Schäfer, J. Treusch, and R. G. Ulbrich, Phys. Rev. Lett. 49, 1281 (1982).

${ }^{10}$ M. Nagai and M. Kuwata-Gonokami, J. Lumin. 100, 233 (2002).

${ }^{11}$ Excitons at High Density, edited by H. Haken and S. Nikitine (Springer, Berlin, 1975).

${ }^{12}$ S. A. Moskalenko and D. W. Snoke, Bose-Einstein Condensation of Excitons and Biexcitons (Cambridge University Press, Cambridge, UK, 2000).

${ }^{13}$ H. Souma, T. Goto, T. Ohta, and M. Ueta, J. Phys. Soc. Jpn. 29, 697 (1970)

${ }^{14}$ Y. Yamada, C. Sasaki, S. Kurai, T. Taguchi, T. Sugahara, K. Nishino, and S. Sakai, J. Appl. Phys. 86, 7186 (1999).
${ }^{15}$ R. Zimmermann, A. Euteneuer, J. Möbius, D. Weber, M. R. Hofmann, W. W. Rühle, E. O. Göbel, B. K. Meyer, H. Amano, and I. Akasaki, Phys. Rev. B 56, R12722 (1997).

${ }^{16}$ V. Bougrov, M. Levinshtein, S. Rumyantsev, and A. Zubrilov, in Properties of Advanced Semiconductor Materials: GaN, AlN, InN, BN, SiC, SiGe, edited by M. E. Levinshtein, S. L. Rumyantsev, and M. S. Shur (Wiley, New York, 2001), p. 2.

${ }^{17}$ G. Göbel, Appl. Phys. Lett. 24, 492 (1974).

${ }^{18}$ H. Yoshida, H. Saito, S. Shionoya, and V. B. Timofeev, Solid State Commun. 33, 161 (1980); H. Yoshida, H. Saito, and S. Shionoya, Phys. Status Solidi B 104, 331 (1981).

${ }^{19}$ When the EHP is created, the exciton state is no longer stable and the excitonic character is thought to be sufficiently small. Therefore, the electron and hole distributions are not affected by the excitonic effect (the Coulomb correlation effect). Thus, we used the conventional non- $k$-conservation model. Actually, the PL line shape can be fitted well by this model as shown in Figs. 2 and 4.

${ }^{20}$ K. Kornitzer, T. Ebner, K. Thonke, R. Sauer, C. Kirchner, V. Schwegler, M. Kamp, M. Leszczynski, I. Grzegory, and S. Porowski, Phys. Rev. B 60, 1471 (1999).

${ }^{21}$ We measured optical gain spectra of highly excited GaN films using a variable stripe method. The peak energy of the gain spectrum is consistent with the peak in the PL spectrum under the same excitation intensity. Then we concluded that this peak is due to the stimulated emission of EHPs.

${ }^{22}$ J. M. Hvam and E. Ejder, J. Lumin. 12/13, 611 (1976).

${ }^{23}$ In fact the excitonic structure was observed in absorption spectrum at $300 \mathrm{~K}$ in A. J. Fischer, W. Shan, J. J. Song, Y. C. Chang, R. Horning, and B. Goldenberg, Appl. Phys. Lett. 71, 1981 (1997). 